\title{
Quench Simulation in an Integrated Design Environment for Superconducting Magnets
}

\author{
Nikolai Schwerg, Bernhard Auchmann, and Stephan Russenschuck \\ CERN AT/MEL, CH-1211, Geneva 23, Switzerland
}

\begin{abstract}
The electrical integrity of superconducting magnets that go through a resistive transition (quench) is an important consideration in magnet design. Numerical quench simulation leads to a coupled thermodynamic and electromagnetic problem, due to the mutual dependence of material parameters. While many tools treat the electromagnetic field problem and the thermodynamic one independently, more recent developments adopt a strongly coupled approach in a 3-D finite-element environment. We introduce a computationally efficient weak electromagnetic-thermodynamic coupling within an integrated design environment for superconducting magnets.
\end{abstract}

Index Terms_Electromagnetic fields, quench simulation, superconducting accelerator magnets.

\section{INTRODUCTION}

$\mathbf{S}^{\mathrm{t}}$ UPERCONDUCTING accelerator magnets operate close to the critical surface of the wire, in order to achieve maximum fields in an economical way. Quench simulation deals with the resistive transition of the superconductor, when the current density, the temperature, or the magnetic induction exceed critical values. The current decay in the magnet is then determined by an external circuit, comprising a protection diode and an energy extraction system (dump resistor). Ohmic heating in the resistive zone results in a propagation of the quench throughout the superconducting coil and rises the voltage above a detection threshold that is used to trigger protection circuits with so-called quench heaters. These heaters cause a resistive transition in adjacent conductors, thus ensuring that the stored energy is dissipated evenly over a larger coil volume. Eddy currents losses in the conductor further increase the cable temperature and accelerate the quench propagation-a process that is referred to as quench-back. A quench simulation tool must, thus, be able to deal with the following:

1) the electromagnetic behavior of the magnet, including the nonlinear magnetization of the iron yoke, eddy-currents in the Rutherford-type cables, as well as the superconductor magnetization;

2) the thermodynamic effects of cooling and quench propagation determined by highly nonlinear material properties (heat capacity, magneto-resistance);

3) the electrical-circuit behavior of the magnet.

As critical parameters, we identify the peak voltage and peak temperature during a quench, as well as the voltage signal of a quench in so-called voltage taps connected to the conductors. The latter is important for the design of quench protection electronics, whereas the former are concerned with the electrical integrity of the magnet. In the light of new challenges, such as fast-ramping and high-field magnets, we stress that these parameters must be considered from the earliest stages of magnet design and optimisation.

Over the past decades, quench simulation routines were developed that decoupled the electromagnetic field problem from

Digital Object Identifier 10.1109/TMAG.2007.916304

Color versions of one or more of the figures in this paper are available online at http://ieeexplore.ieee.org. the thermal simulation. As examples, we mention semi-analytical approaches as in [1]-[3], and a model using a commercial network solver to compute the behaviour of a thermal and an electrical network [4]. A FEM model for the calculation of quench propagation was used in [5] and [6]. The limitations of these methods are reached when strong saturation effects in the yoke occur, which cannot be represented by equivalent inductances and scaled field-maps.

A strong coupling of field calculation, thermal simulation, and electric-circuit analysis is presented in [7] for solenoidal magnets. The thermal problem is solved on the same finite-element mesh as the electromagnetic problem. The required meshing of the coil, air domain, and iron yoke makes this approach computationally expensive for the use in an integrated design process of accelerator magnets, in particular for so-called $\cos \Theta$ magnets made from Rutherford-type conductors.

\section{QUENCH Simulation With ROXIE}

The CERN field computation program ROXIE is a tool for the integrated design of superconducting accelerator magnets [8]. Coils are represented by a set of line-currents, modelling the superconducting strands. Design parameters for the coil and yoke geometries can be addressed in a mathematical optimisation. The field in the yoke is calculated with the coupling method of boundary- and finite elements (BEM-FEM coupling). Only the yoke needs to be meshed.

The electromagnetic field problem and the thermal network exhibit different time constants. Moreover, a BEM-FEM cycle is computationally more expensive than the solution of the coupled electrical-network equations. Thus, we introduce two distinct time stepping loops, as shown in Fig. 1. The quench algorithm is discussed in detail in the following sections.

\section{A. Numerical Field Computation}

The local field distribution in the coil is calculated by means of the BEM-FEM coupling method. At this stage of development, we assume that a magnet is long, compared to the diameter of the magnet aperture (generally true for dipole- and quadrupole magnets in accelerators) and, thus, numerical field computation can be carried out in two dimensions. Eddy-current losses in the cable are calculated from the local field sweep. We distinguish so-called interfilament- and interstrand coupling currents. Interfilament coupling currents are induced in the twisted superconductor-copper matrix of a strand. Interstrand coupling currents are induced in a Rutherford-type 


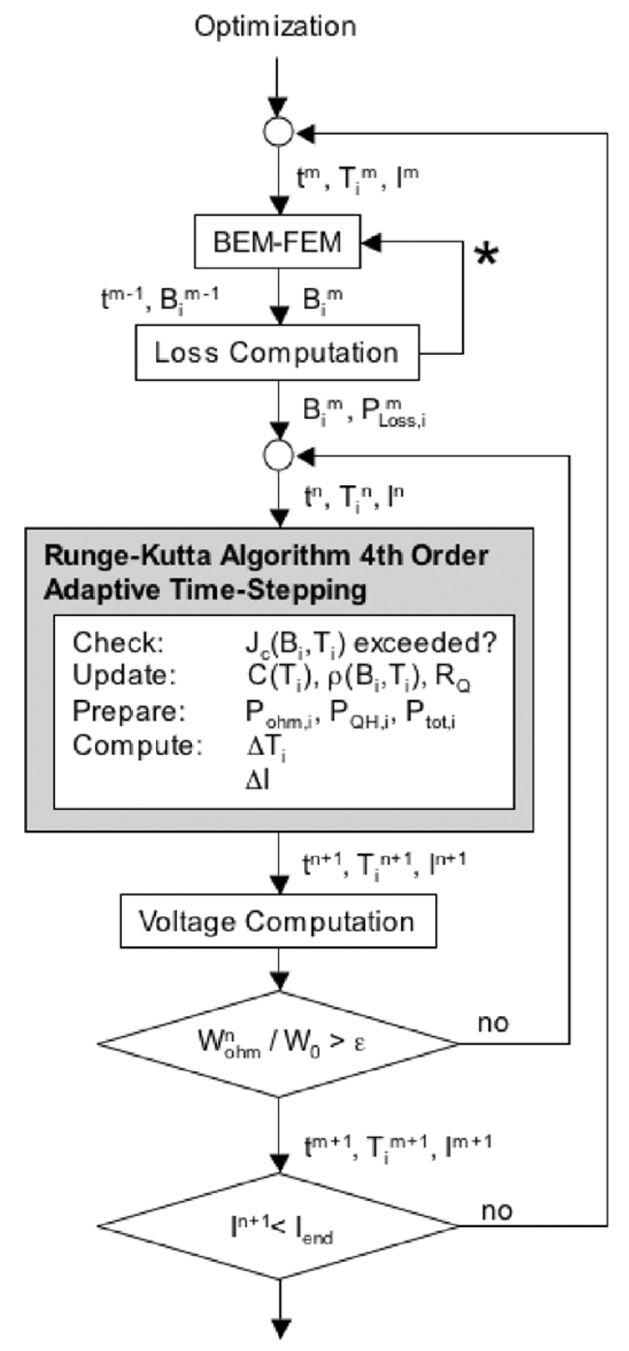

Fig. 1. Block diagram of the ROXIE quench simulation algorithm. We distinguish an outer loop of magnetic field computation and an inner loop of quench simulation.

cable in loops of superconducting strands and contact resistances between strands. The coupling-current time-constants are influenced by the copper resistivity, contact resistances, and geometric parameters, all of which are input parameters to the simulation. The BEM-FEM field model and the coupling-current models are weakly coupled by an iteration loop [9], marked in Fig. 1 with a star.

\section{B. Quench Module}

As illustrated in Fig. 1, the quench module uses the field distribution in the coil from the numerical field computation. It needs to be recalculated at time intervals which are automatically determined, see Section II-C.

The time steps of the inner loop are determined by a fourthorder Runge-Kutta algorithm with adaptive step-size control and an explicit time-integration scheme.

The origin of the simulated quench is designated by the user. Before the quench, the magnet is considered to be in steady state operation at nominal current.

1) Resistive Transition and Resistivity: For each conductor in the cross section we store its working point in terms of current density, average temperature, and peak field. In the super- conducting state, the working point is below the so-called critical surface, which is given by a fit-function $J_{\mathrm{c}}(B, T)$ [10]. At every time step, the conductor's temperature margin to the critical surface is evaluated for given peak-field and current density. ${ }^{1}$ The conductor quenches at zero margin. Current-sharing is neglected.

The copper-resistivity is calculated as a function of temperature and average magnetic induction across the conductor (magneto-resistance). The residual resistivity ratio (RRR) is a measured input parameter to this fit. Furthermore, the copper-to-superconductor ratio and the fit-parameters of the critical surface are input parameters to the quench model.

2) Dissipated Power: The heating power in each conductor is the sum of ohmic losses (current flowing in the copper matrix in a quenched conductor), coupling-current losses and quench heater power, as well as beam losses or other external heating mechanisms. The time constant of the coupling-currents is taken into account.

Coupling-current losses are neglected once the respective conductor has quenched, whereas ohmic losses only set in after a quench has occurred. Quench heaters are considered as circuits that discharge their stored energy upon an external trigger from the quench-protection system. The stored heater-energy and the slope of the discharge (constant, exponential) are user-supplied parameters. The thermal coupling is considered as ideal.

3) Thermal Model: Every conductor constitutes a node in a thermal network. The continuous heat-balance equation

$$
\rho c(T) \frac{\mathrm{d} T}{\mathrm{~d} t}=p+\operatorname{div}(\kappa(T) \operatorname{grad} T)
$$

is solved for every node. Here, $\rho$ denotes the mass density, $c(T)$ the nonlinear specific heat capacity, $\kappa(T)$ the nonlinear and anisotropic thermal conductivity, and $p$ the power density.

In the current state of implementation, heat transfer is considered only between neighbouring cables across the broad side of the cable. The discrete heat-balance equation for the $i$ th conductor, thus, reads

$$
\frac{\mathrm{d} T_{i}}{\mathrm{~d} t}=\frac{1}{\rho_{i} C\left(T_{i}\right)}\left(P_{i}-K\left(T_{i+1}-2 T_{i}+T_{i-1}\right)\right)
$$

where $C\left(T_{i}\right)$ is the mean heat capacity of the conductor, and $P_{i}$ is the sum of dissipated power. Another user supplied parameter is the heat-transfer coefficient $K$, which determines the turn-toturn quench-propagation velocity.

4) Electrical Network: The magnet is connected to an external network that consists of a current source, a bypass-diode with threshold voltage $U_{\mathrm{TH}}$ and forward resistance $R_{\mathrm{D}}$, and possibly a protection resistor $R_{\mathrm{P}}$ in series, compare Fig. 2 . The magnet itself is represented in the network by the resistance $R_{\mathrm{Q}}(t)$ and the differential inductance

$$
L_{\mathrm{d}}(I)=\frac{\mathrm{d} \Psi}{\mathrm{d} I}
$$

where $\Psi$ denotes the linked flux in the magnet coils. The differential inductance depends on the excitation current due to the nonlinear magnetisation of the iron yoke.

When the magnet quenches, the resistivity $R_{\mathrm{Q}}(t)$ rises and the terminal voltage increases. Once the terminal voltage reaches the threshold voltage of the bypass-diode, the diode

\footnotetext{
${ }^{1}$ The nonlinear equation is solved by a Newton algorithm.
} 


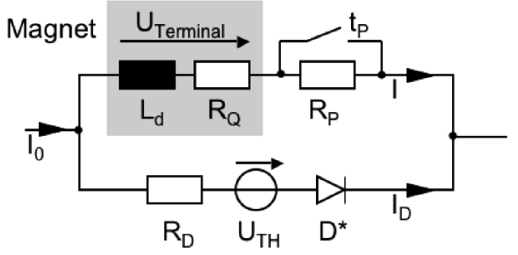

Fig. 2. Electrical network model of a superconducting magnet with protection circuits (protection resistor, bypass diode).

branch becomes conductive and the current commutes from the magnet into the diode. The voltage can be monitored between any pair of conductors. Reaching a threshold triggers the quench heaters and a protection resistor. The electrical network equation reads

$$
\frac{\mathrm{d} I}{\mathrm{~d} t}=-\frac{\left(R_{\mathrm{Q}}(t)+R_{\mathrm{P}}(t)+R_{\mathrm{D}}\right) I-U_{\mathrm{TH}}}{L_{\mathrm{d}}(I)} .
$$

5) Runge-Kutta With Adaptive Time-Stepping: The thermalnetwork-quation (2) and the electrical-network (4) are solved with the classical fourth-order Runge-Kutta algorithm in an explicit time-integration scheme. For each Runge-Kutta time-step, four steps are calculated at different time intervals. A quality-factor [11] indicates if the Runge-Kutta time-step has been appropriate or whether it should be shortened and repeated. If possible, the time-step size is increased for the subsequent step. Adaptive time-stepping is necessary due to the highly-nonlinear material parameters, especially the heat capacity at cryogenic temperatures. It is also needed to resolve the growth of the resistive zone inside the magnet, and the switching-in of the protection resistor. Material parameters need to be updated in every time step.

6) Voltage Calculation: The induced voltage is calculated in all turns of the coil from the time-derivative of the linked flux. To evaluate resistive voltages we interpolate the resistivities before- and after each Runge-Kutta step. The potential to ground of each conductor, and the terminal voltage are calculated by summing all internal voltages in topological order according to the coil-winding scheme.

\section{Loop Termination}

Ohmic losses and coupling-current losses are driven by the stored magnetic energy in the magnet. We use this to calculate the energy decrease in the magnetic field. When it has decreased by a user-supplied factor, the field is updated, i.e., the quench-simulation loop is interrupted and a BEM-FEM time step is carried out. The quench simulation then proceeds with updated values for the field- and coupling-current-loss distribution. In Fig. 4, the differential-inductance graph $\left(L_{\mathrm{d}}\right)$ exhibits the BEM-FEM steps.

\section{RESULTS}

A single aperture dipole magnet similar to those tested in the 1990s at the CERN coil-test-facility is used as the model problem. The magnet employs $\mathrm{Nb}-\mathrm{Ti}$ superconductor technology. It consists of six coil-blocks per quadrant, surrounded by an iron yoke as shown in Fig. 3. The heat transfer between adjacent turns is assumed to be $0.1 \mathrm{~W} / \mathrm{K}$ and the RRR-value of
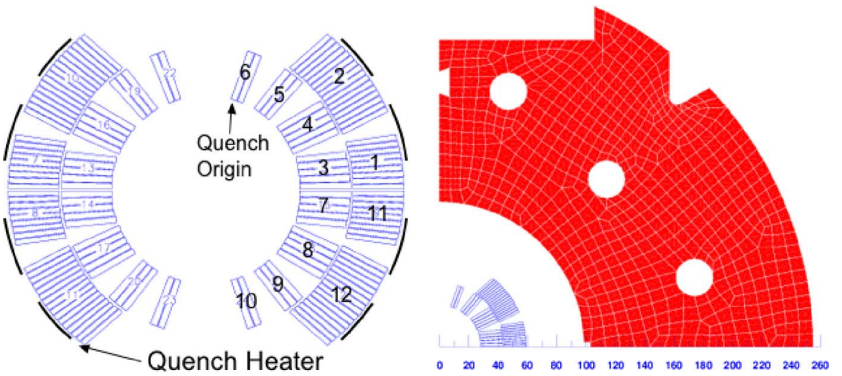

Fig. 3. Model problem for quench simulation. Left: Coil cross section with block numbering and quench heaters. Right: Quadrant of coil and yoke geometry.

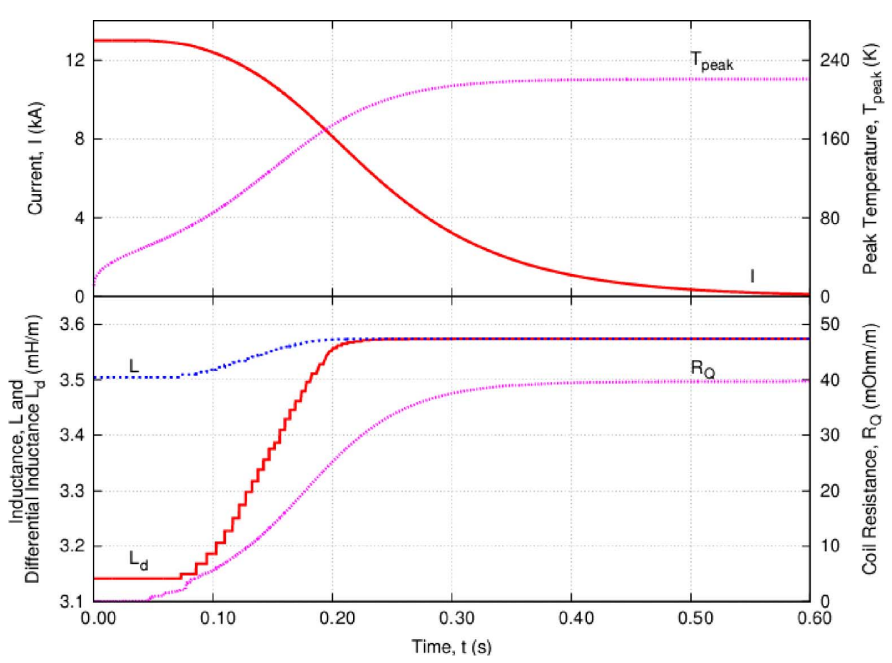

Fig. 4. Top: Magnet current $I$ and peak temperature $T_{\text {peak }}$ during a quench. Bottom: Coil resistance $R_{\mathrm{Q}}$, inductance $L$ and differential inductance $L_{\mathrm{d}}$ as a function of time.

the copper matrix is 150 . With a current of $13 \mathrm{kA}$, this magnets reaches a central field of $9.24 \mathrm{~T}$ at $1.9 \mathrm{~K}$ operation temperature. These are the initial conditions for the quench simulation.

A quench is initiated in the inner-most turn of block 6 by increasing the temperature above the critical temperature. Quench detection is assumed to be immediate, quench heaters are fired with a delay of $40 \mathrm{~ms}$. They contribute $10 \mathrm{~W} / \mathrm{m}$ heating power (decaying with a time constant of $70 \mathrm{~ms}$ ) in every conductor that is covered by a heater, see Fig. 3 .

Fig. 4 (top) shows the development of the temperature $\left(T_{\text {peak }}\right)$ in the conductor where the quench originated, as well as the current decrease. The lower part displays the growth of the coil resistance $R_{\mathrm{Q}}$ and the change of the self inductance $L$ and the differential self inductance $L_{\mathrm{d}}$.

Fig. 5 depicts the temperature margin to quench as a function of time, for all conductors of blocks 1 to 12, compare Fig. 3 (left). Three main features of the quench algorithm are demonstrated.

1) The quench heaters are fired at $t=40 \mathrm{~ms}$. Conductors covered by heaters do not quench simultaneously, due to the inhomogeneous field distribution in the coil.

2) The inner-layer blocks 3-10 quench due to the induced losses (quench-back).

3) The conductors in the outer layer quench due to transversal heat transfer. 


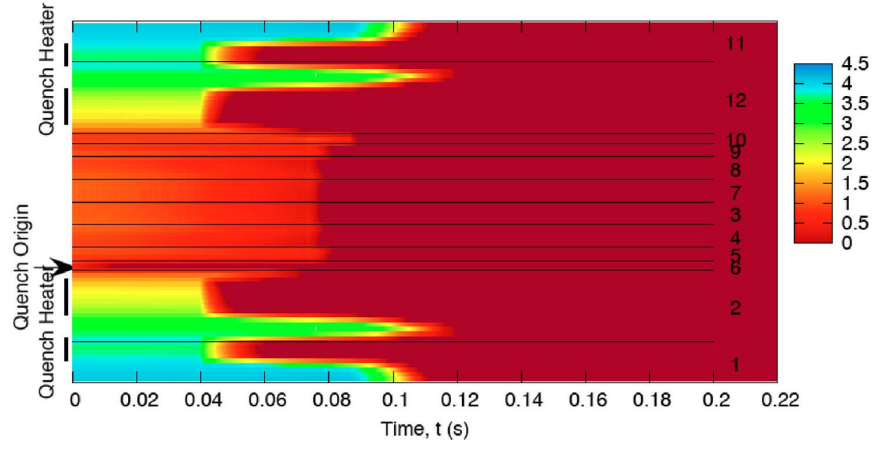

Fig. 5. Temperature margin to quench (in Kelvins) for all conductors in blocks 1 to 12 , compare Fig. 3 (left). Transversal quench propagation due to heat transfer as well as quench-back due to losses can be seen.

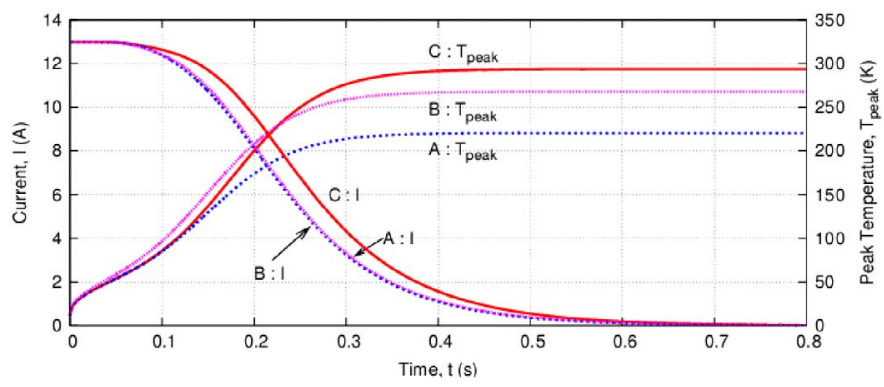

Fig. 6. Magnet current $I$ and peak temperature $T_{\text {peak }}$ for the study of features of the simulation program. Case A uses the full feature set described in Section II-B, with a RRR of 150. Case B uses all features of case A without induced losses, and with a RRR of 80 . Case $\mathrm{C}$ uses a linear inductance, $L$, and a RRR of 150 . It neglects quench-back and heat conduction.

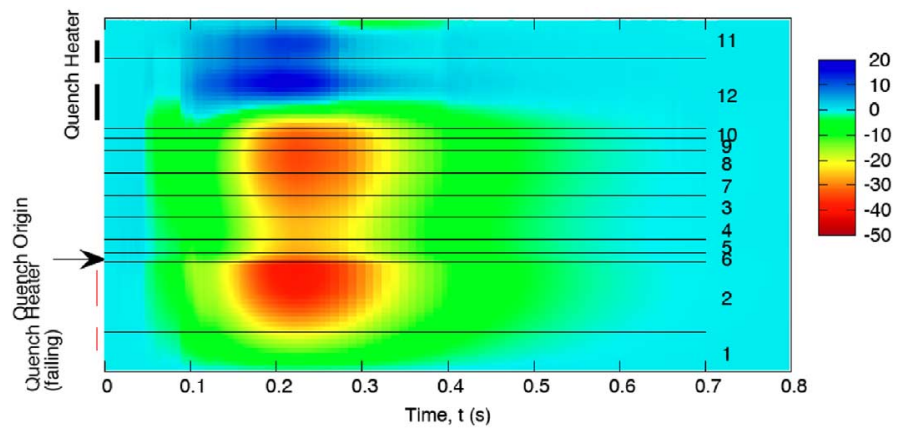

Fig. 7. Electric potential of turns in the superconducting coil (in volts per meter of magnet length) for all conductors in blocks 1 to 12, compare Fig. 3 (left). Only the quench heaters on the lower coil are excited.

Fig. 6 compares the quench model with different feature sets. Case A uses the full feature set described in Section II-B, with a RRR of 150. Case B uses all features of case A without induced losses, and with a RRR of 80 . The results for a model without quench-back, differential inductance, and heat conduction, with a RRR of 150, are shown in case $C$. The current decay in cases $A$ and $B$ is identical. Nevertheless the peak temperature differs by $45 \mathrm{~K}$. Case $\mathrm{C}$ shows a slower current decay and a higher peak temperature as compared to case A.

Fig. 7 shows the distribution of the electric potential in the conductors of blocks 1-12 during a quench. We have simulated a heater failure in the upper half-plane. The voltage is highest in the part of the coil not protected by quench heaters (large potential differences between conductors). Although the turn to turn voltage per meter magnet length is in the range of a few volts per meter, the interlayer voltage between blocks 7 and 11 is of the order of $50 \mathrm{~V}$.

\section{CONCLUSION}

We have implemented a quench simulation algorithm in the CERN field-computation program ROXIE. The approach allows to analyse the coupled electromagnetic and thermodynamic problems. The main structure of the code is established and validated with the simulation of a quench in single-aperture dipole model. It has to be emphasised that the quench algorithm depends on a number of empirical parameters which include the RRR of the copper, the contact resistances of the strands in the cable, the quench heater delay, and the quench detection threshold, among others. Thus, within the physical range of these parameters different sets can be found such that given measurements, i.e., the current decay curve can be fully reproduced. For this reason we have omitted the direct comparison to measurements and defer them to future work [12]. The implementation of the algorithm within the integrated design environment of the ROXIE code allows the systematic study of the sensitivity to these parameters. It is now easy to study the effect of missing quench heaters and to compare different coil and yoke designs for future magnet projects.

\section{REFERENCES}

[1] M. N. Wilson, "Computer simulation of the quenching of a superconducting magnet," Rutherford High Energy Lab., vol. 151, 1968.

[2] M. N. Wilson, Superconducting Magnets. Oxford, U.K.: Oxford Univ. Press, 1983.

[3] M. Canali and L. Rossi, "Dynque: A computer code for quench simulation," in Adiabatic Multicoil Superconducting Solenoids. INFN-TC-93-06, 1993.

[4] D. Hagedorn and F. Rodriguez-Mateos, "Modeling of the quenching process in complex superconducting magnet systems," IEEE Trans. Magn., vol. 28, no. 1, pp. 366-369, Jan. 1992.

[5] F. Sonnemann, "Resistive transition and protection of LHC superconducting cables and magnets," Ph.D. dissertation, Rheinisch-Westfälische Technische Hochschule, Aachen, Germany, 2001.

[6] S. Caspi et al., "Calculating quench propagation with ansys," IEEE Trans. Appl. Superconduct., vol. 13, no. 2, pp. 1714-1717, Jun. 2003.

[7] G. J. C. Aird, J. Simkin, S. C. Taylor, C. W. Trowbridge, and E. Xu, "Coupled transient thermal and electromagnetic finite element simulation of quench in superconducting magnets," presented at the ICAP, Chamonix, France, 2006

[8] ROXIE: Routine for the Optimization of Magnet X-Sections, Inverse Field Calculation and Coil End Design, S. Russenschuck, Ed. Geneva, Switzerland: CERN, Apr. 1999.

[9] B. Auchmann, R. de Maria, S. Kurz, and S. Russenschuck, "2-D electromagnetic model of fast-ramping superconducting magnets," presented at the ICAP, Chamonix, France, 2006.

[10] L. Bottura, "A practical fit for the critical surface of Nb-Ti," IEEE Trans. Appl. Superconduct., vol. 10, no. 1, pp. 1054-1057, Mar. 2000.

[11] L. Collatz, Numerische Behandlung von Differentialgleichungen. Berlin, Germany: Springer, 1955.

[12] N. Schwerg, B. Auchmann, and S. Russenschuck, "Validation of a coupled thermal-electromagnetic quench model for accelerator magnets," IEEE Trans. Appl. Superconduct., to be published.

Manuscript received June 24, 2007. Corresponding author: N. Schwerg (e-mail: nikolai.schwerg@cern.ch). 